\title{
The association of host age and gender with inflammation around neurocysticercosis cysts
}

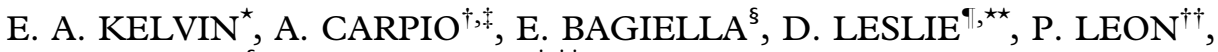 \\ H. ANDREWS ${ }^{\S}$, W. A. HAUSER ${ }^{\ddagger}$, and THE ECUADORIAN \\ NEUROCYSTICERCOSIS GROUP ${ }^{\S \S}$ \\ *HIV Center for Clinical and Behavioral Studies at the New York State Psychiatric Institute and \\ Columbia University, 722 West 168th Street, New York, NY 10032, U.S.A. \\ ${ }^{\dagger}$ School of Medicine, University of Cuenca, Avenida 12 de Abril s/n, P.O. Box 0101-719, \\ Cuenca, Ecuador \\ ${ }^{\star}$ G. H. Sergievsky Center, College of Physicians and Surgeons, Columbia University, 630 West \\ 168th Street, New York, NY 10032, U.S.A. \\ ${ }^{\S}$ Department of Biostatistics, Mailman School of Public Health, Columbia University, 722 West \\ 168th Street, New York, NY 10032, U.S.A. \\ "MRI Diagnostics of Westchester, 503 Grasslands Road, Valhalla, NY 10595, U.S.A. \\ ${ }^{\star \star}$ Good Samaritan Hospital, 255 Lafayette Avenue, Suffern, NY 10901, U.S.A. \\ ${ }^{\dagger}$ Instituto de Diagnóstico por Imágenes, Inés Salcedo 1-91 y A. Cueva, Cuenca, Ecuador \\ *Department of Epidemiology, Mailman School of Public Health, Columbia University, 722 West \\ 168th Street, New York, NY 10032, U.S.A., and Department of Neurology, College of Physicians \\ and Surgeons, Columbia University, 632 West 168th Street, New York, NY 10032, U.S.A.
}

Received 26 August 2008, Revised 30 fune 2009,

Accepted 3 fuly 2009

\begin{abstract}
The results of previous investigations indicate that age and gender may influence the strength of the human host's immune response to infection of the central nervous system with the larvae of Taenia solium. Most of the relevant research on such neurocysticercosis (NCC) has, however, been conducted on hospital-based samples in developing countries, where differential access to healthcare may bias the study results. Using data from 171 NCC patients participating in a treatment trial, the associations of patient age and gender with the presence of inflammation around NCC cysts (i.e. cysts in the transitional phase) have recently been explored, after controlling for measures of economic and geographical access to healthcare. Data on cysts were collected from computed-tomography or magnetic-resonance images taken at four time-points, from baseline to 12-months post-treatment. The odds of having transitional cysts were evaluated by logistic regression whereas Poisson regression was used to explore the numbers of transitional cysts, with generalised estimating equations (GEE) used to account for the multiple observations over time.

After controlling for healthcare access, the odds of having transitional cysts were found to be 1.5-fold higher for the female patients than for the male, although this association was not statistically significant $(P=0.136)$. In the Poisson model, however, the number of transitional cysts was found to be 1.8 -fold higher in the female patients than in the male, and this gender effect was not only statistically significant $(P=0.002)$ but also constant over time. The association of host age with transitional cysts was more complicated, with significant interaction between age and time. It therefore appears that there are significant gender and age differences in the local immune response to NCC, even after adjusting for differences in healthcare access.
\end{abstract}

Reprint requests to: W. A. Hauser, G. H. Sergievsky Center, College of Physicians and Surgeons, Columbia University, 630 West 168th Street, New York, NY 10032, U.S.A.

E-mail: wahauser@optonline.net; fax: +1 2123052518.

${ }^{\S \S}$ The members of the Ecuadorian Neurocysticercosis Group are Drs N. Lisanti (Comprehensive Epilepsy Center, Cuenca), R. Aguirre ('Teodoro Maldonado' Hospital, Guayaquil), M. Serrano ('Carlos Andrade Marín' Hospital, Quito), J. Pesantes ('Eugenio Espejo' Hospital, Quito), J. Moncayo ('Eugenio Espejo' Hospital, Quito) and M. Roman ('Baca Ortiz' Hospital, Quito). 
Human neurocysticercosis (NCC) results from the infection of the central nervous system (CNS) with the larval stage of the intestinal pork tapeworm, Taenia solium. Humans may develop the disease after ingesting eggs excreted by someone harbouring an adult tapeworm in their intestines (Singhi and Ray, 1999). NCC is possibly the most heterogeneous infection of the CNS, with a wide variety of clinical manifestations, including seizures, headaches, psychiatric disorders, and focal neurological deficits (White, 1997). The clinical manifestations are generally thought to be related to the number, size and location of the parasitic cysts, and the host's immune response to the infection (Schantz and Kramer, 1995; White, 1997). The mechanisms that underlie the variety in host reaction to infection of the CNS are, however, not well understood (Del Brutto et al., 1991), the wide diversity of symptoms indicating a complex hostparasite-environment relationship.

Most symptomatic NCC infections appear to occur among people in their mid-adult years (Carpio, 2002). Although there does not seem to be any gender difference in the general prevalence of symptomatic NCC (Sciutto et al., 2000), it has been suggested that both age and gender influence the strength of the host's immune response. The severe encephalitic form of parenchymal NCC, which manifests as overwhelming inflammation, appears to be more common among women and children than in men (López-Hernández and Garaizar, 1982; Earnest et al., 1987; Del Brutto and Sotelo, 1988; Garcia and del Brutto, 2000). In two studies, women have also been found to exhibit a stronger immune response to CNS infection with T. solium than men (Del Brutto et al., 1988; Fleury et al., 2004). One of those studies also found that adult NCC cases aged $>48$ years had fewer leucocytes in their CSF than younger cases (Fleury et al., 2004). In addition, younger adults seem to present more often with single transitional cysts, which are characterised by local oedema, than older adults (Fleury et al., 2004: Sáenz et al., 2006).

There has been some concern that some, if not all, of the reported associations between host age and/or gender and the clinical manifestations of NCC are the result of bias in the selection of subjects (Thurn, 1988; Carpio, 2002). Since the definitive diagnosis of NCC requires computed tomography (CT) or magnetic resonance imaging (MRI), the vast majority of research on the disease has been conducted on hospitalbased samples. Such samples are susceptible to selection bias (Carpio, 2002), especially in developing countries, where regional or economic differences in access to healthcare, as well as frequent migration from areas where NCC is highly endemic to areas of low endemicity, might bias any comparisons made within the hospital-based sample. Patients of a certain age or gender may, for example, have relatively poor (economic and/or physical) access to healthcare because of a weak health infrastructure, and may therefore only present, for diagnosis and treatment, when the symptoms of their disease become severe. Such patients may not be representative of NCC cases in general.

The main aim of the present study was to examine, within a hospital-based population in Ecuador, the influence of host age and gender on the host's local immune response to CNS infection with $T$. solium, as measured by the presence of inflammation around the cysts. Possible associations between a patient's age and gender and his or her immune response were explored after adjusting for potential confounders, including factors that may be associated with access to healthcare.

\section{PATIENTS AND METHODS}

The data for the analyses described here came from a clinical trial of treatment for symptomatic NCC in which patients were 
randomized to receive treatment of their symptoms plus either albendazole or placebo. The detailed study design, as well as a flow chart depicting participation at each step of the study, has already been published (Carpio et al., 2008) but a brief description follows.

\section{Patients}

Patients were recruited from six hospitals in Ecuador - three in Quito, two in Cuenca, and one in Guayaquil. Patients were eligible to participate in the study if they presented within 2 months of the (new) onset of symptoms associated with NCC and if CT or MRI revealed that they had active and/or transitional NCC cysts. Patients who only had calcified cysts and those who were pregnant, had active tuberculosis, syphilis, papilloedema, ocular cysticercosis, active ulcers, or some other progressive and lifethreatening disorder were excluded. Patients who had received treatment for NCC during the past year or who had undergone systemic treatment with steroids within 30 days of presentation were also ineligible for the study and, about halfway through the study, patients with ventricular shunt were excluded, for safety reasons.

Between January 2001 and February 2003, 178 NCC patients agreed to participate in the study and were randomized for treatment. No data were collected on the six patients who dropped out before receiving treatment, and the data from another patient, who, although treated, was subsequently determined to be ineligible for the study, were excluded from the final analyses. The 171 NCC patients included in the data analysis were aged 3-82 years (mean $=40$ years) and $92(54 \%)$ were male. The participants were of primarily lower socio-economic status. Almost half (49\%) had never attended secondary school - a proportion similar to the Ecuadorian national value (World Bank, 2006) — and $31 \%$ had a monthly household income of $<$ U.S. $\$ 70$.

\section{Data Collection}

The brain of each patient was examined by CT or MRI at baseline (no more than 2 weeks before treatment with albendazole or placebo) and 1, 6 and 12 months posttreatment. The cysts detected were classified according to their location in the brain and their phase of evolution. Cysts containing a live or viable parasite were classified as active. Those showing obvious signs of a host immune response (i.e. inflammation; see below) and in which the parasite was degenerating were classified as transitional, whereas calcified lesions in which the parasite was dead were classified as inactive (Carpio et al., 1994). Each of the images was read independently by two neuroradiologists, one in Ecuador and one in the U.S.A.; the inter-rater reliability of the readings was adequate, with kappas, for identifying that a patient had cysts in a specific location or phase, ranging from 0.4 0.7 . The readings of just one neuroradiologist (the one based in the U.S.A) were used for the present analyses.

Transitional cysts were identified on CT or MRI by the presence of a variable amount of vasogenic or white-matter oedema surrounding the cyst. Such oedema appears as a hypodense area on standard CT and as a hyperintense area on T2-weighted MRI, with irregular borders that follow the white-matter tracks. On contrast-enhanced CT or MRI, each transitional cyst shows enhancement of the cyst wall, which initially appears as a ring but becomes a nodule as the cyst collapses. Transitional cysts, defined by the presence of inflammation around the cysts, were the main outcome considered in the data analyses.

Information about the patient's gender and age, as well as information about potential confounders, was collected at baseline. Overall, six variables were considered as potential confounders that might be related to transitional cysts. Three of these potential confounders - the patient's monthly household income, the distance of the patient's residence from an asphalt road 
$(\mathrm{km})$, and the presence/absence of the patient's residence in the same province as the treating hospital - were used as indicators for economic and physical, infrastructure-related barriers to healthcare access. Unequal access to healthcare in Ecuador may mean that some groups seek care only when symptoms are severe, and, in NCC, symptom severity is related to the case's immune response. The patient's monthly household income was a measure of the patient's ability to pay for, and therefore receive, healthcare. Information on this variable was collected in three categories: low income (<U.S. $\$ 70 /$ month), middle income (U.S.\$70-200/month) and high income (>U.S.\$200/month). The distance of the patient's residence from an asphalt road may influence geographical access to healthcare facilities. The location of the patient's residence in the same province as the treating hospital may be another indication of geographical access to healthcare and/or an indicator of migration status, both of which may influence healthcare access.

The other three potential confounders considered may be associated with the outcome, transitional cysts, or with the investigators' ability to identify such cysts: the type of imaging done (CT or MRI); the total number of cysts in all phases; and the drug treatment (albendazole or placebo). Type of image (CT or MRI) was considered a potential confounder because, since transitional cysts are easier to identify by MRI than by CT (Garcia et al., 2003), measurement error rates will differ depending on which type of image is being read. The total number of cysts is a potential confounder because the more cysts a patient has, the higher the probability that one or more will be in the transitional phase. In addition, even though the two NCC 'treatments' were randomly assigned, drug assignment was considered a potential confounder because of its presumed influence on the progress of NCC cysts through the three main phases in their evolution. Finally, as outcome in each patient was measured at four different timepoints and the cyst phase varies over time, time (measured in months from baseline) was also included in all the data models.

\section{Ethics}

An informed-consent form for study participation was signed by all participants or, in the case of participants aged $<18$ years, by one of their legal guardians. Participants aged $<18$ years also provided oral assent for participation. Approval for the study was granted by the Columbia University Institutional Review Board, the Office for Protection from Research Risks (OPRR) of the U.S. National Institutes of Health, and the ethics committee of each participating hospital in Ecuador.

\section{Statistical Analysis}

Data were analysed using the SAS 9.0 software package (SAS Institute, Cary, NC). The outcome, transitional cysts, was modelled over the four time-points (baseline and 1,6 and 12 months post-treatment). Transitional cysts were examined in total, for all locations in the brain, before those within the parenchymal and extraparenchymal regions (intraventricular and subarachnoid) were explored separately. The small numbers involved prevented the influence of host age-group on the extraparenchymal transitional cysts to be investigated.

The outcome was modelled as a dichotomous variable (presence/absence of transitional cysts) using logistic regression, and as a count variable (number of transitional cysts) using Poisson regression. Generalised estimating equations (GEE) were used to account for the multiple observations of each patient over time.

The effect of age and gender was explored after controlling for time, and the interaction of age and gender with time was investigated, to determine if the effects found were constant over time. Patient observations in which the brain image showed too many cysts to count were 
excluded from the analyses. This criterion excluded 14 observations at baseline, and 12,13 and 11 observations at 1,6 and 12 months post-treatment, respectively. Because of missing data on some of the confounders and this exclusion of participants with too many cysts to count, the final sample size for the regression models ranged from 154 to 157 participants.

As the distribution of the number of transitional cysts by age was not linear, age categories were created for the analyses. Although age was divided approximately into quintiles, the age category 3-19 years was maintained, even though it contained only about $10 \%$ of the patients, because subjects aged $<20$ years are considered to be at relatively low risk of CNS infection with T. solium (Carpio, 2002) but at relatively high risk of severe NCC (LópezHernández and Garaizar, 1982; Earnest et al., 1987; Del Brutto and Sotelo, 1988; Garcia and del Brutto, 2000).

\section{Steps in Defining the Final Regression Models}

Four steps were used in determining the final regression models for presentation.

\section{INITIAL, UNADJUSTED MODELS}

Initially, the regression models were run unadjusted for the potential confounders, with time (baseline or 1,6 or 12 months post-treatment), patient gender and patient age as the independent variables. As previously noted, age was not included when extraparenchymal transitional cysts were the outcome considered (because the sample size was too small for including four agedummy variables).

\section{ASSESSING CONFOUNDING}

Each of the six potential confounders was then added separately to the initial models. If the beta values for either gender or age changed by $10 \%$ or more with the addition of a potential confounder, that confounder was considered to be sufficiently important to be included in the final model. Using this criterion, in some cases a potential confounder was determined to be an important confounder in one model but not in another. The confounders included in each final model therefore differed (they are specified in the table footnotes). The odds ratios (OR) and rate ratios (RR) presented in Table 2 are the results from the final models adjusted for all the important confounders.

\section{ASSESSING INTERACTION WITH TIME}

Once the final adjusted models had been established, interaction terms were added to assess if the association between gender and the outcome, or that between age and the outcome, varied significantly over time. The $P$-values for interaction with time presented in Table 2 are the $P$-values for these interaction terms.

\section{MODELS STRATIFIED ON TIME}

In order to describe the interaction with time, the adjusted models were also run, for transitional cysts in all locations, separately for each time-point (i.e. stratified on time). Figures 1 and 2, which show the OR and $\mathrm{RR}$ for age (the variable for which there was a statistically significant interaction) stratified on time, help to assess the direction of the interaction between age and time.

\section{Sensitivity Analyses}

Because the data being analysed were longitudinal, there was concern that missing observations - caused by missed interim visits, death, drop-out, or total loss to follow-up - might influence the results. Therefore, after determining the final models, the influence that the missing observations might have was explored through a series of sensitivity analyses, where the missing data were given values according to various assumptions. For the 'mildest' assumptions tested, the datum for a missing observation was given the same value as the 
corresponding value recorded at a previous or subsequent time-point. The models were also run under more extreme assumptions, assigning either the maximum number of transitional cysts possible (all cysts being transitional) or zero transitional cysts (the minimum possible) to all the missing observations. As none of the resultant data models gave results that were markedly different from those seen when only the valid data were used, the findings of these sensitivity models are not presented or discussed below.

\section{RESULTS}

\section{Distribution of the Transitional Cysts}

At baseline, the 157 patients in the study who did not have too many cysts to count (14 patients had too many cysts to count at baseline) had a total of 1100 NCC cysts. Among these patients, 98 had cysts in the transitional phase, totalling 270 such cysts. Eighty-five of the patients with transitional cysts had such cysts in their brain parenchyma (with a total of 207 parenchymal transitional cysts) and 22 had transitional cysts in the extraparenchymal regions of their brains (with a total of 56 extraparenchymal cysts). Five patients had a total of seven transitional cysts in indeterminable locations in their brains and these seven cysts were only counted in the analyses looking at all locations combined. Three of these five patients also had parenchymal transitional cysts.

Table 1 shows the distribution of transitional cysts by patient gender and age. A higher proportion of the female patients than of the male had transitional cysts at each time-point, and the female patients also had a higher mean number of transitional cysts at each time-point, although neither of these gender-related differences appeared to be statistically significant. Compared with the other age-groups considered, the patients aged 3-19 years were those most likely to have transitional cysts at baseline and 1 month post-treatment and those least likely to have such cysts at 6 and 12 months post-treatment, although only the baseline difference was statistically significant. Patients aged 3-19 years had the lowest mean number of transitional cysts at all four time-points but this difference never reached statistical significance.

\section{Regression Results}

CYSTS IN ALL LOCATIONS WITHIN THE BRAIN After adjusting for confounders, the odds of having transitional cysts were found to be higher for the female patients than the male, although the association was not statistically significant $(\mathrm{OR}=1.5 ; P=0.136)$. The female patients did, however, have a significantly higher mean number of transitional cysts than the male. Specifically, the female patients were found to have, on average, 1.8-fold more transitional cysts than their male counterparts $(P=0.002)$, after controlling for time and other confounders. There was no interaction between time and gender in predicting either the presence/absence or number of transitional cysts (Table 2).

Although the odds of having transitional cysts were higher for the patients aged 5082 years than for any of the younger agegroups, only the patients aged 20-29 years were significantly less likely to have such cysts than the patients aged 50-82 years $(\mathrm{OR}=0.4 ; P=0.042)$. For patients age $3-19$ years there was a significant interaction with time $(P=0.003)$ in predicting the odds of having transitional cysts. Figure 1 shows the adjusted OR for each age-group, stratified by time. The patients aged 3-19 years had the highest OR of any age-group at baseline and 1 month post-treatment but the lowest OR at both 6 and 12 months posttreatment. In the Poisson-regression models, the patients aged 3-19 years were found to have significantly fewer transitional cysts than the patients aged 50-82 years $(\mathrm{RR}=0.3 ; P<0.001)$. Although the other age-groups were not significantly associated with the number of transitional cysts, the 


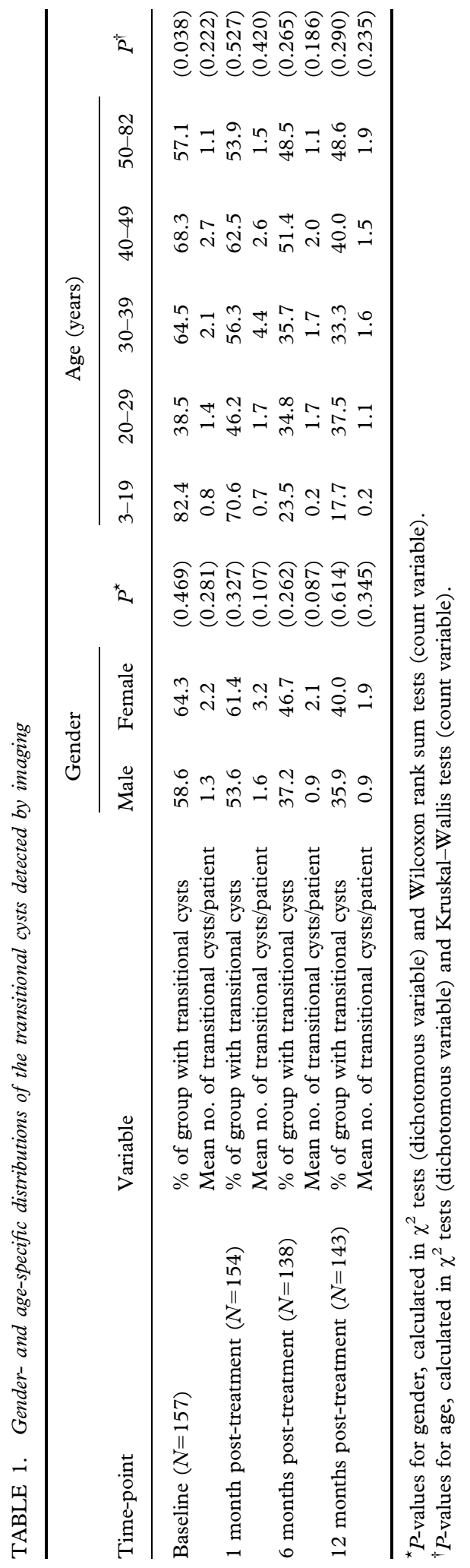




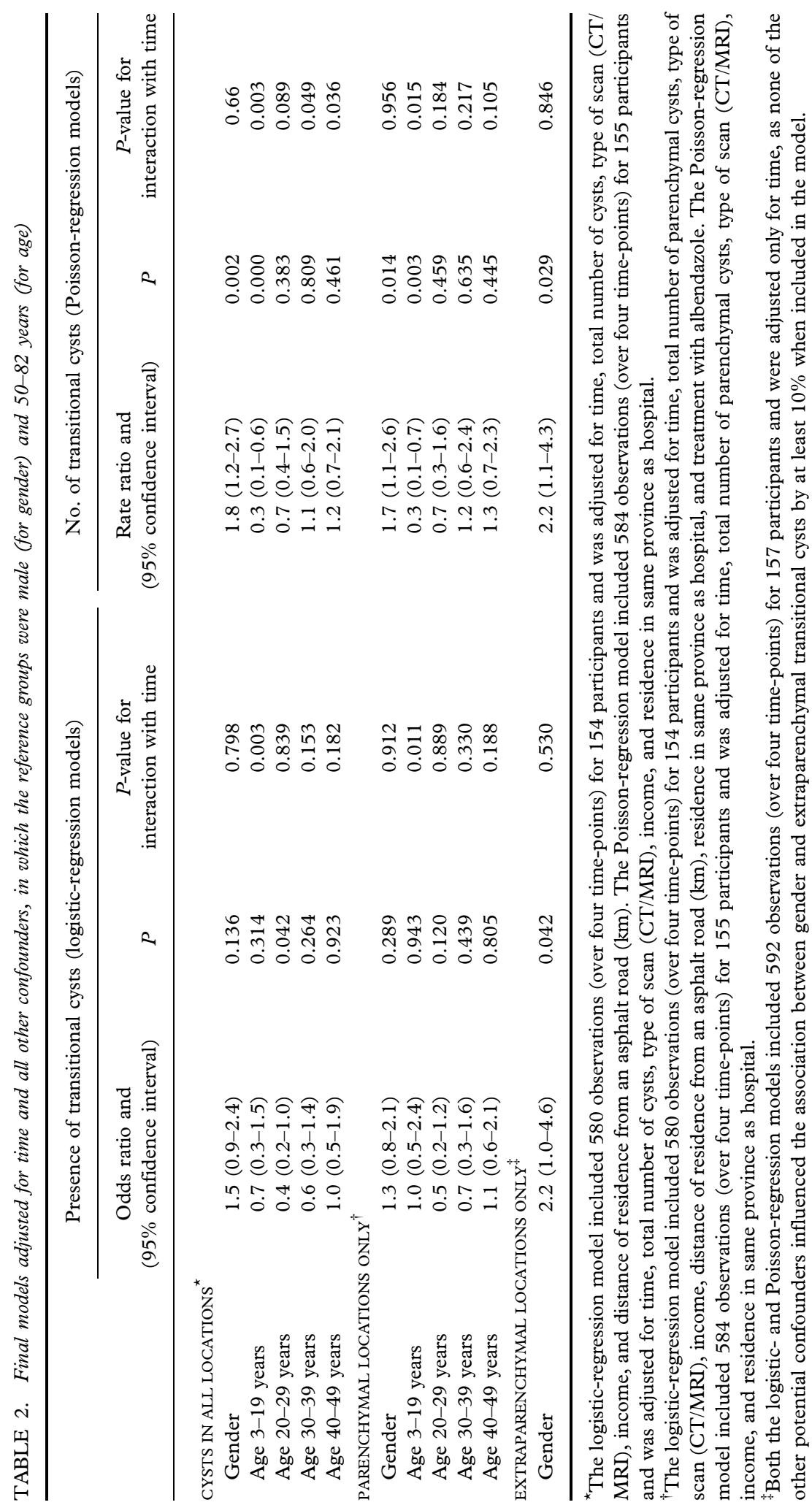




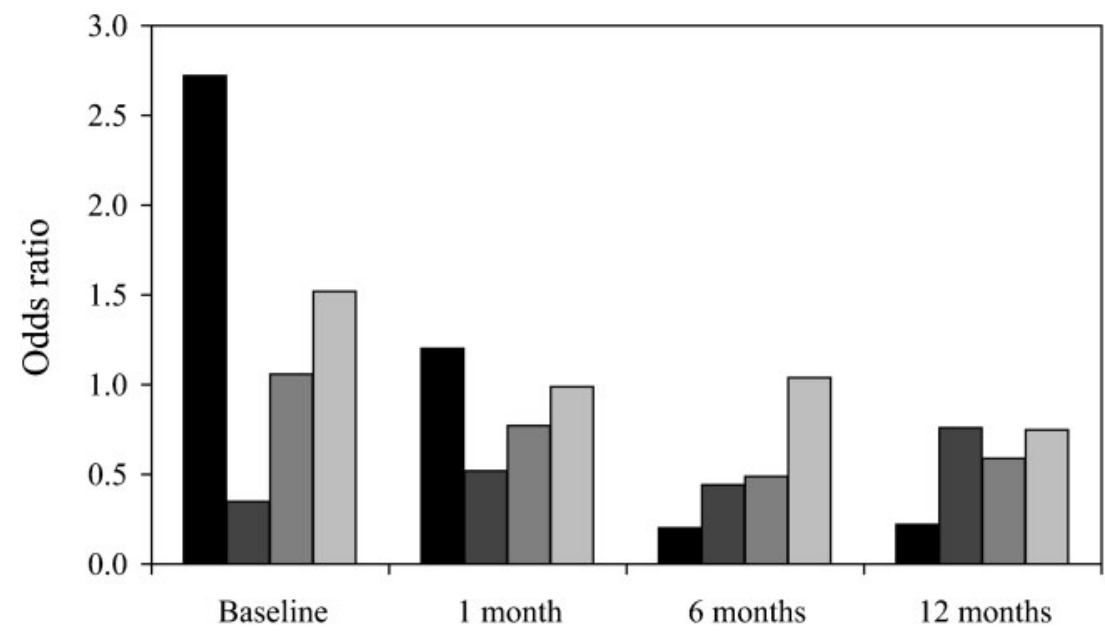

Time-point

FIG. 1. Stratified logistic-regression models: adjusted odds ratios for the presence of transitional cysts in all

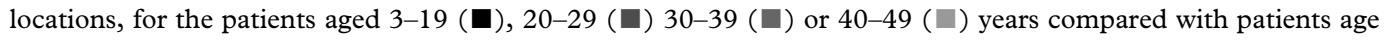
50-82 years, at each time-point. At baseline $(N=154)$, the odds ratios and $(95 \%$ confidence intervals) for those aged 3-19, 20-29, 30-39 and 40-49 years were $2.7(0.5-13.7), 0.4(0.1-1.1), 1.1(0.4-3.1)$, and 1.5 (0.5-4.1), respectively. The corresponding values changed to $1.2(0.6-2.5), 0.5(0.2-1.6), 0.8(0.3-2.3)$ and $1.0(0.3-3.1)$ at 1 month post-treatment $(N=151), 0.2(0.1-0.8), 0.4(0.1-1.6), 0.5(0.1-1.7)$ and $1.0(0.1-3.3)$ at 6 months posttreatment $(N=135)$, and $0.2(0.1-0.9), 0.8(0.2-2.5), 0.6(0.2-2.0)$ and $0.8(0.3-2.1)$ at 12 months post-treatment $(N=140))$.

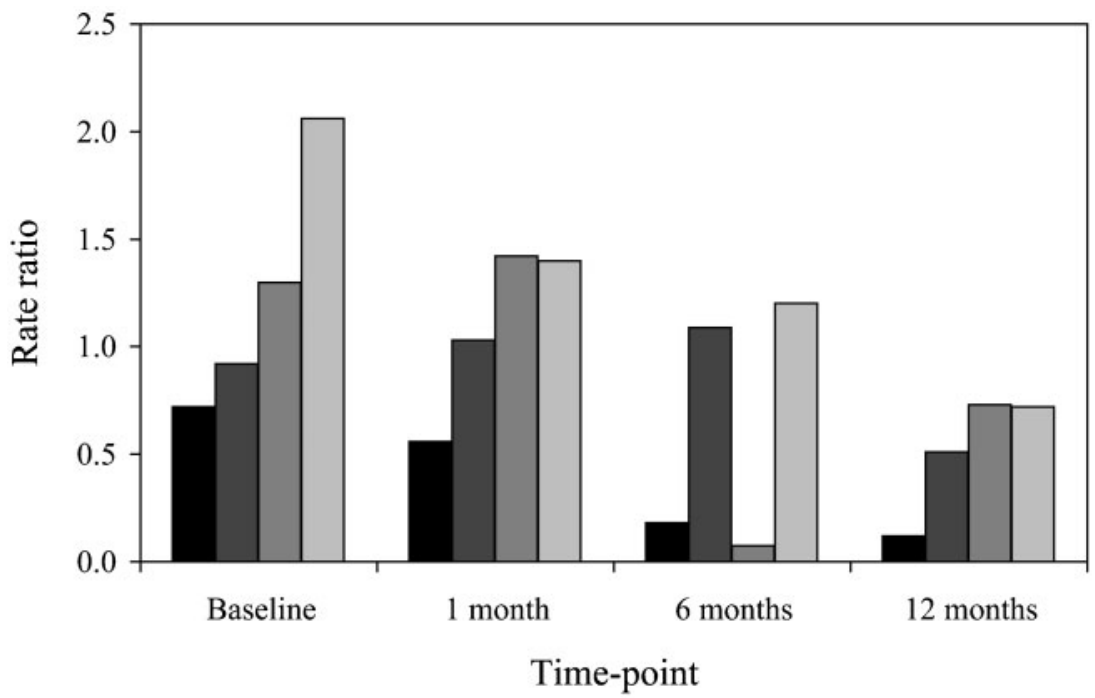

FIG. 2. Stratified Poisson-regression models: adjusted rate ratios for the number of transitional cysts in all

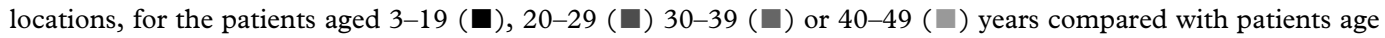
$50-82$ years, at each time. At baseline $(N=155)$, the rate ratios and ( $95 \%$ confidence intervals) for those aged $3-19$, 20-29, 30-39 and 40-49 years were $0.7(0.4-1.4), 0.9(0.4-2.0), 1.3(0.7-2.4)$ and $2.1(1.2-3.6)$, respectively. The corresponding values changed to $0.6(0.3-1.0), 1.0(0.5-2.1), 1.4(0.7-2.8)$ and $1.4(0.8-2.0)$ at 1 month posttreatment $(N=152), 0.2(0.1-0.5), 1.1(0.6-2.2), 0.1(0.0-1.4)$ and $1.2(0.7-2.1)$ at 6 months post-treatment $(N=136)$, and $0.1(0.0-1.4), 0.5(0.2-1.2), 0.7(0.4-1.4)$, and $0.7(0.4-1.2)$ at 12 months post-treatment $(N=141)$. 
interaction of age and time in the Poisson model was statistically significant, or almost statistically significant $(P=0.089$ for the patients aged 20-29 years), in all agegroups. Figure 2 shows the RR for each of the younger age-groups compared with the patients aged 50-82 years, stratified on time. Although patients in the youngest age-group had the lowest $R R$ at each of the four time-points, their RR decreased over time.

\section{CYSTS IN PARENCHYMAL BRAIN}

In the adjusted logistic-regression models looking at parenchymal transitional cysts, again there was no significant association found between gender or age, but there was a significant interaction with time for patients aged $3-19$ years $(P=0.011)$. In the adjusted Poisson model, the female patients were found to have significantly more transitional cysts in parenchymal locations than the male patients $(\mathrm{RR}=1.7 ; \mathrm{P}=0.014)$, and the patients aged 3-19 years had significantly fewer transitional cysts in such locations than the patients aged $50-82$ years $(\mathrm{RR}=0.3 ; P=0.003)$. That the interaction of age with time was statistically significant for the comparison between the patients aged 3-19 years and the patients aged $50-82$ years $(P=0.015)$ indicates, however, that the corresponding $\mathrm{RR}$ was not constant over time. Neither gender nor the other agegroups demonstrated such an interaction with time (Table 2).

\section{CYSTS IN EXTRAPARENCHYMAL BRAIN}

In the adjusted logistic-regression model for extraparenchymal transitional cysts, the female patients were found to be significantly more likely to have transitional extraparenchymal cysts than the male $(\mathrm{OR}=2.2 ; P=0.042)$. In the corresponding Poisson model, the female patients were also found to have significantly more extraparenchymal transitional cysts, on average, than the male $(\mathrm{RR}=2.2 ; P=0.029)$.

\section{DISCUSSION}

Gender and age differences in immune response have been demonstrated for several diseases, although the associations are complicated and vary in direction depending on the infectious agent being examined (Morales-Montor et al., 2004). It has also been suggested that age-related differences in immune response are actually related to physiological processes such as sexual maturation (Remoué et al., 2001). Thus one might expect to find age-related differences in diseases for which there are genderrelated differences.

Looking at helminth infections, female mice have frequently been found to be more susceptible to experimental infection with T. crassiceps than male mice (Huerta et al., 1992; Bojalil et al., 1993; Larralde et al., 1995; Fragoso et al., 1998). Curiously, such infection leads to the increased feminization of both male and female mice, with increases in oestradiol in all the mice and a decrease in testosterone in the male mice (Larralde et al., 1995). With T. solium, castration and pregnancy have been found to increase the prevalence of cysticercosis in pigs, presumably through changes in the concentrations of some sex hormones (Morales et al., 2002), and histological damage to $T$. solium cysts in pigs vaccinated against the parasite was found to be greater in the older hosts than in the younger animals (Huerta et al., 2000). Although age (Fleury et al., 2004; Sáenz et al., 2006) and gender (Del Brutto et al., 1988; Fleury et al., 2004) differences in immune response to $T$. solium infection of the human CNS have also been reported, concern has been raised about the possibility that selection bias is responsible for these apparent differences (Thurn, 1988). In addition, there has been no gender- or age-specific information about how $T$. solium cysts behave over time in the human CNS.

In the present study, there was clear evidence of both gender and age differences in the patient's immune response to $T$. solium 
infection of the CNS, even after evaluating and controlling for several potential confounders, including barriers to healthcare access. Although the female patients were more likely to have transitional cysts than the male, this association was only statistically significant for the extraparenchymal transitional cysts. On average, however, the female patients also harboured more transitional cysts, in their brains as a whole, in the parenchymal region of their brains, and in the extraparenchymal region of their brains, than the male patients. The strength of this gender-specific association was constant over time, and was greater for the extraparenchymal cysts than for the parenchymal. It appears, therefore, that, in humans, females mount a stronger local immune response to the presence of $T$. solium cysts in their brains than males who have similar infections. Although this should lead to a faster clearance of viable $T$. solium in women than in men, it may also place women at greater risk of developing the more severe symptoms of NCC. This may explain why the incidence of the encephalitic form of parenchymal NCC is greater in female cases of NCC than in male cases (López-Hernández and Garaizar, 1982; Earnest et al., 1987; Del Brutto and Sotelo, 1988; Garcia and del Brutto, 2000).

The relationship between transitional cysts and the age of the NCC case seems to be somewhat more complicated. In the present study, patients in the youngest agegroup (3-19 years) were not (as might have been expected from the literature on NCC) significantly more likely to have transitional cysts than the patients in the oldest agegroup (50-82 years), and they harboured significantly fewer transitional cysts than the older patients. There were, however, significant age-time interactions when transitional cysts were explored as either a dichotomous or count variable. Thus, the strength of the age-specific association was not constant over time. Specifically, compared with the oldest age-group (50-82 years), the odds ratio for having transitional cysts was highest for the patients aged 3-19 years (i.e. higher than for any of the other age-groups considered) at baseline and 1 month post-treatment but lowest (i.e. lower than for any of the other age-groups considered) for this youngest age-group at 6 and 12 months post-treatment. This change over time is primarily attributable to the rapid decrease, over the 12 months of follow-up, in the proportion of patients in the youngest age-group who had transitional cysts. While $82 \%$ of the patients aged 3-19 years had transitional cysts at baseline, only $17.7 \%$ of the same group of patients had transitional cysts when examined about 12 months later. In comparison, the proportion of patients in the oldest age-group (aged 50 82 years) found to have transitional only fell from $57.1 \%$ to $48.6 \%$ over the same period (Table 1). The observed interaction with time may therefore be an indication that the cysts go through the transitional phase at different rates, depending on the patient's age. Alternatively, it might be an indication that, despite controlling for several potential barriers to healthcare access, some selection bias still occurred in the present study and that patients of different ages seek treatment at different points in the evolution of their NCC. In the latter case, the observed variation with time may indicate that the patients of different ages investigated in the present study were recruited at varying times in their disease process.

The present results indicate that, in humans, there are significant gender- and age-specific differences in the local immune response to CNS infection with $T$. solium, these differences remaining significant even after adjusting for differences in healthcare access that may be important causes of selection bias when using hospital-based samples. Although these findings were not influenced by loss to follow-up or other causes of missing data, the present study did suffer from several other weaknesses. Firstly, the study included only 171 patients and thus the statistical power may have been 
insufficient for some of the models. The fact that, in general, gender and age were found to be better at predicting the number of transitional cysts than the odds of having transitional cysts probably reflects the difference in statistical power when examining an outcome as a dichotomous variable instead of a count variable. Secondly, the study cohort only included 22 patients with transitional cysts in extraparenchymal locations, precluding a valid examination of the association of age category with such extraparenchymal transitional cysts. Thirdly, no information about the NCC patients who attended the participating hospitals but who were ineligible for the study (including those who simply refused to participate) was collected. This information would have been helpful in determining if the study participants were representative of the NCC cases attending the hospitals (e.g. in terms of household income or location of residence). Another weakness of the study may have been the reliability of the CT or MRI readings. While the inter-rater reliability appeared adequate, with kappa values of $0.4-0.7$, it was certainly not perfect, indicating some misclassification that could further decrease the study's statistical power. Finally, the present patients were only followed for 12 months. Later scans would have been useful in determining if the associations and trends found in the present analyses continue, or if there are other ageor gender-specific differences in the patient's immune response to CNS infection with $T$. solium over time periods beyond 12 months.

In future research, additional host factors that may influence the immune response to CNS infection with $T$. solium, and the effects of these host factors over a period exceeding 12 months, should be explored. In addition, the relationship between the human host's immune response and their clinical symptoms should be better described, as this may be an important cause of bias when studying hospital-based populations.
It is important to take into account possible barriers to healthcare access when conducting research in hospital-based populations. If proxies for selection bias, such as income and location of residence, are measured during the study, then they can be controlled for in the final analyses. These proxies for selection bias are not confounders in the traditional sense (i.e. alternate causes of the outcome) but they can still be controlled for if they are identified and measured, and doing this should increase confidence in the results of studies using hospital-based populations.

ACKNOWLEDGEMENTS. This study was supported by the U.S. National Institute of Neurological Disorders and Stroke (via grant R01-NS39403), and the time the senior author (E.A.K.) spent working on the analyses described was supported by a Ruth L. Kirschstein National Research Service Award (grant 5F31NS051946). Glaxo/SKB and Acromax Co. supplied the albendazole and placebo. The authors are indebted to Drs E. Castro, G. Rodríguez, I. Reinoso, V. Paredes and C. Barrionuevo, all of the 'Carlos Andrade Marín' Hospital in Quito, for their support in patient care, and to Dr F. Santillán, from the Social Security Hospital in Cuenca, for patient referrals. Other members of the Neurocysticercosis Clinical Trial Group who collaborated in this study include K. Quinde, A. Montenegro, A. Vasco, M. Obregon, L. Villalta, Dr N. Hernandez and M. A. Garcia.

\section{REFERENCES}

Bojalil, R., Terrazas, L. I., Govezensky, T., Sciutto, E. \& Larralde, C. (1993). Thymus-related cellular immune mechanisms in sex-associated resistance to experimental murine cysticercosis (Taenia crassiceps). Fournal of Parasitology, 79, 384-389.

Carpio, A. (2002). Neurocysticercosis: an update. Lancet Infectious Diseases, 2, 751-762.

Carpio, A., Placencia, M., Santillán, F. \& Escobar, A. (1994). A proposal for classification of 
neurocysticercosis. Canadian Fournal of Neurological Sciences, 21, 43-47.

Carpio, A., Kelvin, E. A., Bagiella, E., Leslie, D., Leon, P., Andrews, H. \& Hauser, W. A. (2008). Effects of albendazole treatment on neurocysticercosis: a randomised controlled trial. Fournal of Neurology, Neurosurgery and Psychiatry, 79, 10501055.

Del Brutto, O. H. \& Sotelo, J. (1988). Neurocysticercosis: an update. Reviews of Infectious Diseases, 10, 1075-1087.

Del Brutto, O. H., Garcia, E., Talámas, O. \& Sotelo, J. (1988). Sex-related severity of inflammation in parenchymal brain cysticercosis. Archives of Internal Medicine, 148, 544-546.

Del Brutto, O. H., Granados, G., Talamas, O., Sotelo, J. \& Gorodezky, C. (1991). Genetic pattern of the HLA system: HLA A, B, C, DR, and DQ antigens in Mexican patients with parenchymal brain cysticercosis. Human Biology, 63, 85-93.

Earnest, M. P., Reller, L. B., Filley, C. M. \& Grek, A. J. (1987). Neurocysticercosis in the United States: 35 cases and a review. Reviews of Infectious Diseases, 9, 961-979.

Fleury, A., Dessein, A., Preux, P. M., Dumas, M., Tapia, G., Larralde, C. \& Sciutto, E. (2004). Symptomatic human neurocysticercosis - age, sex and exposure factors relating with disease heterogeneity. Fournal of Neurology, 251, 830-837.

Fragoso, G., Lamoyi, E., Mellor, A., Lomeli, C., Hernández, M. \& Sciutto, E. (1998). Increased resistance to Taenia crassiceps murine cysticercosis in Qa-2 transgenic mice. Infection and Immunity, 66, 760-764.

Garcia, H. \& del Brutto, O. (2000). Taenia solium cysticercosis. Infectious Disease Clinics of North America, 14, 97-119.

Garcia, H. H., Gonzalez, A. E. \& Gilman, R. H. (2003). Diagnosis, treatment and control of Taenia solium cysticercosis. Current Opinion in Infectious Diseases, 16, 411-419.

Huerta, L., Terrazas, L. I., Sciutto, E. \& Larralde, C. (1992). Immunological mediation of gonadal effects on experimental murine cysticercosis caused by Taenia crassiceps metacestodes. Fournal of Parasitology, 78, 471-476.

Huerta, M., Sciutto, E., García, G., Villalobos, N., Hernández, M., Fragoso, G., Díaz, J., Díaz, A., Ramírez, R., Luna, S., García, J., Aguilar, E., Espinoza, S., Castilla, G., Bobadilla, J. R., Avila, R., José, M. V., Larralde, C. \& de Aluja, A. S. (2000). Vaccination against Taenia solium cysticercosis in underfed rustic pigs of Mexico: roles of age, genetic background and antibody response. Veterinary Parasitology, 90, 209-219.

Larralde, C., Morales, J., Terrazas, I., Govezensky, T. \& Romano, M. C. (1995). Sex hormone changes induced by the parasite lead to feminization of the male host in murine Taenia crassiceps cysticercosis. fournal of Steroid Biochemistry and Molecular Biology, 52, 575-580.

López-Hernández, A. \& Garaizar, C. (1982). Childhood cerebral cysticercosis: clinical features and computed tomographic findings in 89 Mexican children. Canadian Fournal of Neurological Sciences, 9, 401-407.

Morales-Montor, J., Chavarria, A., de León, M. A., del Castillo, L. I., Escobedo, E. G., Sánchez, E. N., Vargas, J. A., Hernández-Flores, M., RomoGonzález, T. \& Larralde, C. (2004). Host gender in parasitic infections of mammals: an evaluation of the female host supremacy paradigm. Fournal of Parasitology, 90, 531-546.

Morales, J., Velasco, T., Tovar, V., Fragoso, G., Fleury, A., Beltrán, C., Villalobos, N., Aluja, A., Rodarte, L. F., Sciutto, E. \& Larralde, C. (2002). Castration and pregnancy of rural pigs significantly increase the prevalence of naturally acquired Taenia solium cysticercosis. Veterinary Parasitology, 108, 4148.

Remoué, F., To Van, D., Schacht, A. M., Picquet, M., Garraud, O., Vercruysse, J., Ly, A., Capron, A. \& Riveau, G. (2001). Gender-dependent specific immune response during chronic human schistosomiasis haematobia. Clinical and Experimental Immunology, 124, 62-68.

Sáenz, B., Ruíz-Garcia, M., Jiménez, E., HernándezAguilar, J., Suastegui, R., Larralde, C., Sciutto, E. \& Fleury, A. (2006). Neurocysticercosis: clinical, radiologic, and inflammatory differences between children and adults. Pediatriac Infectious Disease fournal, 25, 801-803.

Schantz, P. \& Kramer, M. (1995). Larval cestode infections: cysticercosis and enchinococcosis. Current Opinion in Infectious Diseases, 8, 342-350.

Sciutto, E., Fragoso, G., Fleury, A., Laclette, J. P., Sotelo, J., Aluja, A., Vargas, L. \& Larralde, C. (2000). Taenia solium disease in humans and pigs: an ancient parasitosis disease rooted in developing countries and emerging as a major health problem of global dimensions. Microbes and Infection, 2, 18751890.

Singhi, P. \& Ray, M. (1999). Focal seizures with single small ring-enhancing lesion. Seminars in Pediatric Neurology, 6, 196-201.

Thurn, J. R. (1988). Neurocysticercosis and possible sex-related severity of inflammatory reaction. Archives of Internal Medicine, 148, 2689.

White Jr, A. C. (1997). Neurocysticercosis: a major cause of neurological disease worldwide. Clinical Infectious Diseases, 24, 101-115.

World Bank (2006). Ecuador Country Profile. Washington, DC: World Bank. 